\title{
Development of a multivariable improvement measure for gout
}

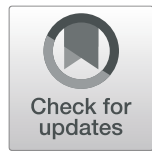

Naomi Schlesinger ${ }^{1}$, N. Lawrence Edwards ${ }^{2}$, Anthony E. Yeo ${ }^{3}$ and Peter E. Lipsky ${ }^{4^{*}}$ (D)

\begin{abstract}
Background: Gout is a heterogeneous inflammatory disease with numerous clinical manifestations. A composite means to assess the impact of therapy on numerous aspects of gout could be useful.

Methods: Results from patients treated with pegloticase or placebo in two randomized clinical trials and their open-label extension were assessed using a novel evidence-based Gout Multivariable Improvement Measure (GMIM) derived from previously reported criteria for remission and complete response. Improvement was defined as serum urate $(\mathrm{s} U)<6 \mathrm{mg} / \mathrm{dL}$ and absence of flares during the preceding 3 months plus 20,50 , and $70 \%$ improvement in tophus size, patient global assessment, pain, and swollen and tender joints.

Results: Patients treated with pegloticase manifested a significantly greater GMIM20, 50, and 70 response vs those treated with placebo (GMIM20 at 6 months $37.1 \%$ vs $0 \%$, respectively). Higher response rates were significantly more frequent in subjects with persistent urate lowering (GMIM 58.1\% at 6 months) in response to pegloticase versus those with only transient urate lowering (GMIM 7.1\% at 6 months). However, when the requirement for a decrease in $\mathrm{SU}$ to $<6 \mathrm{mg} / \mathrm{dL}$ was omitted, a substantial percentage of subjects with transient urate lowering met the GMIM clinical criteria. A sensitivity analysis indicated that gout flares contributed minimally to the model. The response measured by GMIM persisted into the open-level extension for as long as 2 years. Finally, subjects who received placebo in the randomized control trials, but pegloticase in the open-label extension, manifested GMIM responses comparable to that noted with pegloticase-treated subjects in the randomized controlled trials.
\end{abstract}

Conclusions: GMIM captures changes in disease activity in response to treatment with pegloticase and may serve as an evidence-based tool for assessment of responses to other urate-lowering therapies in gout patients.

Keywords: Gout, Disease activity, Pegloticase

\section{Key messages}

What is already known about this subject?

- A Gout Activity Score (GAS) has been proposed, but its utility in clinical trials has not been documented.

- Tools to assess response to gout treatment are needed.

What does this study add?

* Correspondence: peterlipsky@comcast.net

${ }^{4}$ AMPEL BioSolutions, LLC, Charlottesville, VA, USA

Full list of author information is available at the end of the article
- A new Gout Multivariable Improvement Measure (GMIM) was developed based on evidence from previous clinical trials.

- The GMIM captures both biochemical and clinical information and is sensitive to change.

How might this impact on clinical practice or future developments?

- The GMIM may provide a means to assess clinical benefit of urate-lowering therapy in clinical trials and medical care. 


\section{Background}

Inflammatory rheumatic diseases are generally multifaceted disorders, and the complex pathology underlying these conditions makes it difficult to assess patient status and the efficacy of therapy with a single representative outcome measure [1]. The lack of a single gold standard for patient monitoring has prompted the development of composite measures for many rheumatic diseases [2], and there are well-established indices for rheumatoid arthritis [3, 4], psoriatic arthritis [5-7], systemic lupus erythematosus [8-10], ankylosing spondylitis [11], osteoarthritis [12, 13], and fibromyalgia [14].

Gout is also a multifactorial inflammatory disease. Even though the cause of gout, namely hyperuricemia, is known, patients experience a wide range of symptoms, including severe pain, acute and persistent inflammatory arthritis, tophi, and disability associated with both flares and chronic disease $[15,16]$. Moreover, the spectrum of disease changes with time $[17,18]$. It has been recommended that multiple domains should be evaluated when assessing effects of treatment for gout $[19,20]$. Although assessment of urate-lowering therapy (ULT) for this disease has focused primarily on the ability to lower serum urate $(\mathrm{sU})$ and decrease in the frequency of flares [21-25], some trials have included additional endpoints to address effects of treatment on pain, arthritis, and disability $[26,27]$.

The overall goal of ULT is the dissolution of all urate deposits and the prevention of new deposits from occurring. Although it is assumed that this occurs when the $\mathrm{sU}$ level is maintained at a level $<6 \mathrm{mg} / \mathrm{dL}$, there is no direct means other than imaging modalities to assess this. Therefore, there is the general recognition that assessment of ULT and other treatments for gout could be facilitated by endpoints that more closely reflected the multidimensional effects of urate deposition. This has prompted multiple groups to propose composite measures aimed at this goal. Over a decade ago, an Outcome Measures in Rheumatology (OMERACT) special interest group proposed core domains for interventional studies in chronic gout that included sU, gout flare recurrence, tophus regression, joint damage imaging, health-related quality of life, musculoskeletal function, patient global assessment (PGA), ability to participate in usual activities, and safety and tolerability [28]. Since that time, there have been multiple discussions regarding the domains most important for inclusion in a composite measure for use in clinical trials of advanced gout [29]. These measures are generally aimed at determining the patient's status at a given point in time rather than providing a change score that captures response to treatment over time. Despite very high interest and intensive deliberation, development and testing of composite measures for either acute or advanced gout has been very limited. A composite index for evaluation of treatments for acute gout has been developed [30], and a composite Gout Activity Score (GAS) has been shown to be sensitive to change and to have predictive validity with a correlation to the domains of the Gout Impact Scale (GIS) [31, 32]; however, these composite measures have not been tested in trials of ULT.

The present study used results from two identical randomized controlled trials (RCTs) of pegloticase (NCT00325195, NCT01356498) for development of a composite measure for capturing responses to gout treatment. The construction of the Gout Multivariable Improvement Measure (GMIM) involved evaluation of criteria proposed to define remission in gout and included sU, frequency of flares, tophus reduction, pain evaluation, and PGA [33]. Previous evaluation of patient responses added tender and swollen joints to the list of patient features incorporated [34]. GMIM therefore comprised $\mathrm{sU}$, flares, tophi, PGA, pain, and swollen and tender joint counts (SJC, TJC). Improvement was defined as $\mathrm{sU}<6 \mathrm{mg} /$ $\mathrm{dL}$ and absence of flares during the preceding 3 months plus $20 \%, 50 \%$, or $70 \%$ improvement in $\geq 3$ of the other 5 clinical evaluations.

The aim of this study was to use GMIM to assess the degree of response in patients with chronic refractory gout treated with pegloticase and to validate the composite response measure by comparing its ability to discriminate those with persistent urate lowering from pegloticase treatment with those with transient urate lowering and also those receiving placebo. GMIM was also tested in a group of patients who received placebo in the RCTs and began pegloticase in the open-label extension to evaluate the composite measure in a situation not used to develop the tool. Although based on data from a subset of subjects with advanced gout, the tool may have utility in other settings.

\section{Methods \\ Design of pegloticase clinical trials}

The design of the two identical RCTs of pegloticase and their open-label extension (OLE) that provided the data analyzed in this study have been described in detail previously $[26,35]$ and are only briefly summarized here. Both studies received institutional review board approval for each site, and written informed consent and Health Insurance Portability and Accountability Act assurances were completed for each participant prior to enrollment. These trials included adults with chronic refractory gout with one or more of the following: $\mathrm{sU} \geq 8.0 \mathrm{mg} / \mathrm{dL}$ and $\geq 3$ selfreported gout flares during the previous 18 months; $\geq 1$ tophus; chronic gouty arthritis; and failure to respond to the maximum medically appropriate allopurinol dose, as determined by the treating physician, or a contraindication to this drug. Patients were randomized to 6 months of intravenous infusions of either pegloticase $8 \mathrm{mg}$ every 2 
weeks (q2w), every 4 weeks (q4w), or placebo [26]. The primary endpoint for the RCTs was reduction of $\mathrm{sU}(\mathrm{sU}<$ $6.0 \mathrm{mg} / \mathrm{dL}$ ) $\geq 80 \%$ of the time during month 3 (extending from the week-9 infusion to just before the week-13 infusion) and month 6 (extending from the week-21 infusion to the week-25 final study visit). Any patient not achieving this goal or who did not complete the trial was classified as a nonresponder. Patients from the RCTs could continue into the OLE in which they were given the choice of receiving pegloticase $\mathrm{q} 2 \mathrm{w}$ or $\mathrm{q} 4 \mathrm{w}$ [35].

These trials had a large number of secondary endpoints which made results particularly useful for development of a single composite outcome measure, including tophus resolution; reduction in gout flares; decreases in TJC and SJC; PGA of disease activity; and patient-reported changes in pain, physical function (HAQ-Disability Index), and quality of life (36-Item Short Form Health Survey) [26]. Patients were assessed at baseline and at the week 13 and 19 visits as well as at the week 25 final visit for secondary endpoints. In addition, patients were evaluated for up to 27 months of the OLE.

\section{Construction of the Gout Multivariable Improvement Measure (GMIM)}

A total of 85 subjects were treated with biweekly pegloticase. Overall, $42 \%(n=36)$ of the patients who received pegloticase $8 \mathrm{mg}$ every 2 weeks were responders (persistently lowered sU) to treatment in the RCTs. In previous work, responders were evaluated to determine whether they met combined criteria for remission defined by a previous Delphi exercise [33]. In addition, the clinical data from the responders was employed to develop criteria for a complete response. This was derived by employing a repeated measures mixed effects model with backward elimination that related clinical and laboratory changes observed throughout the trial [34]. In this exercise, numerous outcome measures were considered, but sU, PGA, numbers of tender and swollen joints, and the degree of tophus resolution controlling for repeated measures were found to contribute the most information to the complete response model [34]. Based upon the previous criteria for remission and complete response, the final set of criteria employed in GMIM were identified and included $\mathrm{sU}<6 \mathrm{mg} / \mathrm{dL}$, PGA scores, visual analog scale (VAS) pain levels, TJC and SJC, the number of flare episodes, and the degree of tophus area resolution. Improvement was defined as $\mathrm{sU}<$ $6 \mathrm{mg} / \mathrm{dL}$ and the absence of flares during the preceding 3 months plus $20 \%, 50 \%$, or $70 \%$ improvement in $\geq 3$ of the other 5 clinical criteria. Because improvement in $\mathrm{sU}$ and a decrease in flare frequency were uniformly used in clinical trials and were felt to be mandatory components of response, these were required along with a variety of clinical features that might vary in different patients.
Since sU can vary with time, evaluation of clinical responses was also carried out with and without the requirement for achievement of $\mathrm{sU}<6 \mathrm{mg} / \mathrm{dL}$. Sensitivity analysis was done by removing one variable at a time from the analysis.

\section{Statistical analysis}

All comparisons were carried out with Fisher's exact test to determine whether there was a nonrandom association between two variables. Chi-square with correction for continuity was used when the frequency of events was small. Statistical significance was defined as $p<0.05$. Components that contributed the most information to the GMIM model were determined by multiple linear regression with backward elimination of the least statistically significant term to model the relationship between the dependent variable, the GMIM response, and various independent variables.

All calculations were carried out with SAS version 9.4 (Cary, NC).

\section{Results}

GMIM components (i.e., $\mathrm{sU}<6 \mathrm{mg} / \mathrm{dL}$, flares, tophi, PGA, pain, and swollen and tender joints) were determined from previous analyses of the patient data in the RCTs of pegloticase. A combination of criteria from the proposed remission criteria [33] and also criteria for a complete response [34] were considered. Although some criteria contributed little to the complete response model, such as pain and flares [34], they were included in the final GMIM because they were thought to be important in evaluating patients with gout. Moreover, it was felt that the way the data had been collected in the RCTs may have decreased its discriminative value [34]. For example, pain was collected as a general condition and not as related to gout. The previous exercise had evaluated the criteria strictly as a means to evaluate the induction of remission or complete response [34]. Here, we employed these criteria to determine the degree of response. Initially, the entire group of 85 subjects receiving biweekly pegloticase was assessed for the frequency of subjects achieving GMIM20, 50, and 70 status and compared to the outcome of subjects receiving placebo (Fig. 1a, supplementary table 1). No placebo-treated subjects achieved GMIM status, whereas approximately $40 \%$ of pegloticase-treated subjects became GMIM20 responders after the 6-month RCT. Since some pegloticasetreated subjects only had a transient urate-lowering effect, we were interested in determining whether pegloticase might have a clinical benefit even if the contemporaneous $\mathrm{sU}$ was not $<6 \mathrm{mg} / \mathrm{dL}$. To accomplish this, we relaxed the GMIM status by omitting the requirement for a $s \mathrm{U}<6$ $\mathrm{mg} / \mathrm{dL}$. As can be seen in Fig. $1 \mathrm{~b}$ and supplementary table 1, the frequency of GMIM responders was 

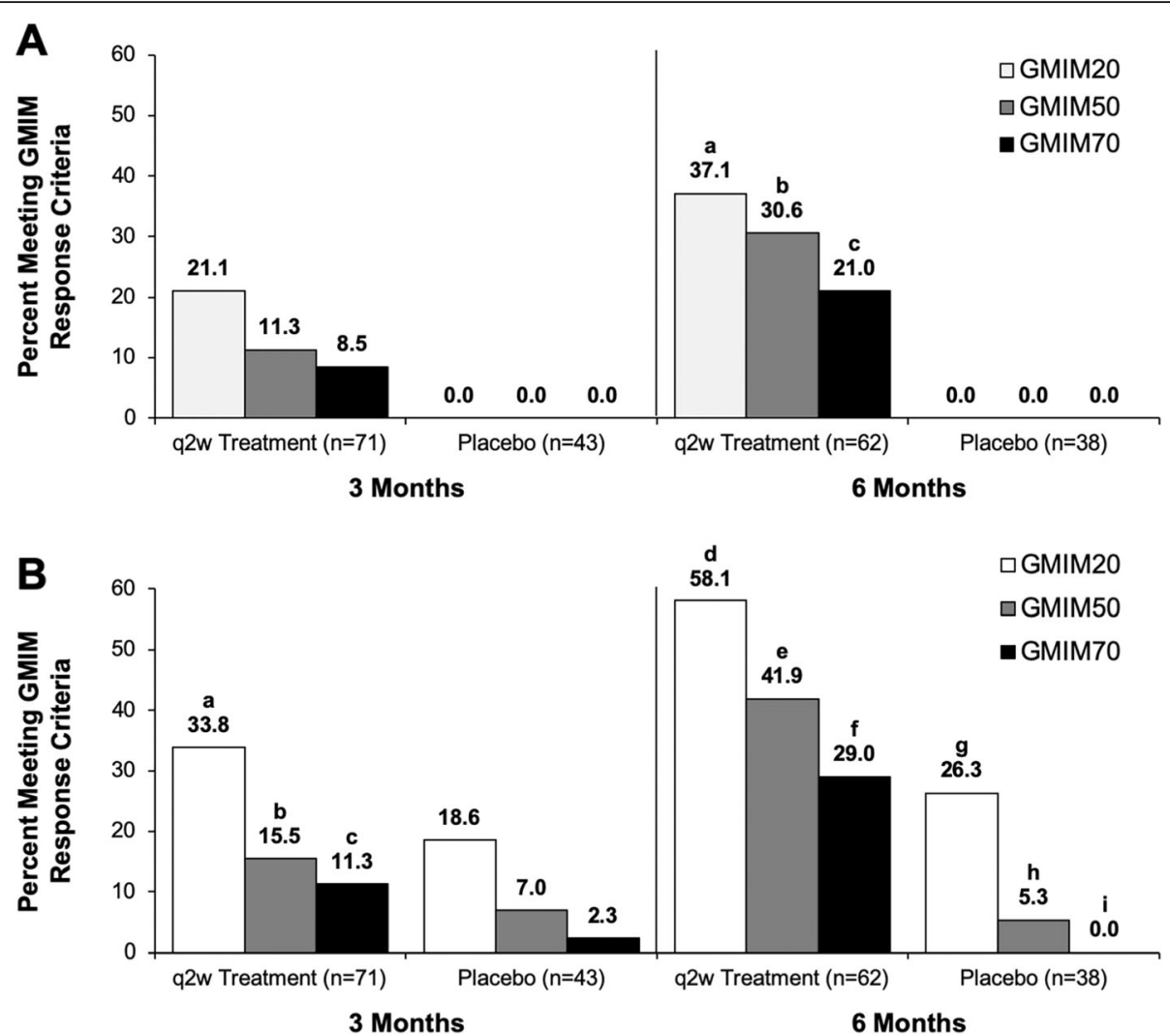

Fig. 1 Percentage of subjects treated with pegloticase q2w or placebo reaching GMIM criteria at 3 and 6 months in the RCTs. Number of evaluable subjects is shown for each group. The percentage of responders is shown above the bars. a Results for all GMIM criteria and $\mathbf{b}$ Results for all criteria except urate $<6.0 \mathrm{mg} / \mathrm{dL}$. The statistical analysis of the data is shown in the supplementary material

considerably higher in the pegloticase-treated subjects when the urate lowering requirement was removed, with approximately $60 \%$ of pegloticase-treated subjects achieving a GMIM20 and 30\% a GMIM70 after 6 months of treatment. However, in this circumstance, nearly $30 \%$ of placebotreated subjects also achieved a GMIM20 by 6 months, but only infrequently achieved a higher level of response.

To explore the relationship between persistent urate lowering caused by pegloticase and the percentage of subjects achieving GMIM responses in greater detail, we divided the pegloticase-treated subjects into those with persistent urate lowering (responders) and those with only transient urate lowering (nonresponders) and analyzed the percentage achieving GMIM responses. Thirtyfour of 85 subjects treated with biweekly pegloticase had persistent urate lowering (responders) and also entered the OLE. As can be seen in Fig. 2 and supplementary table 2, GMIM responder status was mainly limited to the subjects with persistent urate lowering, with $36.1 \%$ and 58.3\% achieving GMIM20 responses by 3 and 6 months, respectively. Less than $10 \%$ of subjects who failed to maintain persistent urate lowering (nonresponders) attained GMIM20 responses. It should be noted that many of these nonresponders still had urate lowering through 3 months of therapy and a small number had urate lowering at 6 months even though they did not meet the strict criteria of being a responder [34].

To explore the clinical response in the nonresponders in greater detail, the criterion for achievement of $\mathrm{sU}<6 \mathrm{mg}$ / $\mathrm{dL}$ was omitted (Fig. 3, supplementary table 3). As with the analysis shown in Fig. 1, this resulted in some subjects in the placebo group achieving GMIM20, 50, and 70 responses at 3 months and GMIM20 and 50 responses at 6 months. There were also increases in GMIM20, 50, and 70 responses at 3 and 6 months for the q2w nonresponders. At 6 months, q2w responders achieved GMIM20 significantly more often vs placebo or nonresponders, whereas the nonresponders exhibited more frequent responses compared to placebo-treated subjects.

\section{Sensitivity analysis}

A sensitivity analysis was carried out by recalculating GMIM20, 50, and 70 for each group with elimination (one at a time) of each of the clinical parameters. The requirement to achieve $\mathrm{sU}<6 \mathrm{mg} / \mathrm{dL}$ was also not required for these analyses (Fig. 4, supplementary table 4). As can be seen, most variables contributed to the model with the exception of flares, whose elimination had little impact on the percentage 


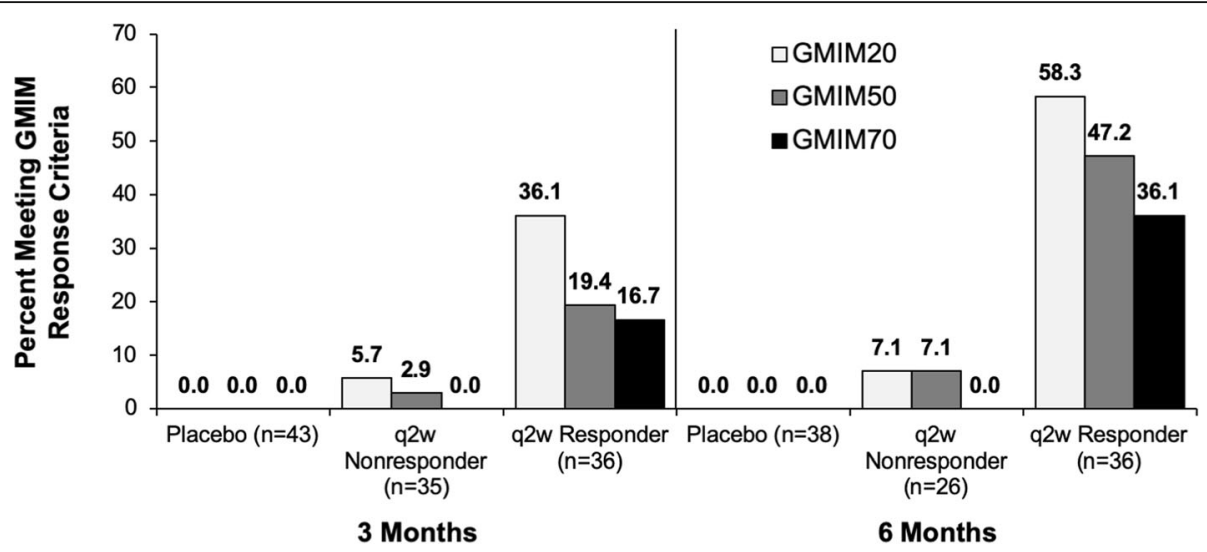

Fig. 2 Percentage of responders and nonresponders to pegloticase or placebo-treated subjects meeting GMIM criteria at 3 and 6 months. Data show percentage of subjects meeting GMIM criteria including serum urate $<6 \mathrm{mg} / \mathrm{dL}$. Number of evaluable subjects is shown for each group. The percentage of responders is shown above the bars. The statistical analysis of the data is shown in the supplementary material

of subjects meeting GMIM criteria. To explore this further, we carried out regression analysis of all of the GMIM components. Multiple linear regression analysis indicated that the components that consistently contributed information to GMIM were sU, PGA, tender joints, pain, and swollen joints. Tophus area and flare contributed only inconsistently. Because only a subset of patients had measurable tophi, we evaluated the performance of GMIM in those subjects independently (Supplementary figure 1). GMIM effectively separated responses in the tophaceous patients. Moreover, eliminating resolution of tophus area as a component of GMIM significantly decreased the measured responses, implying that tophus resolution significantly contributed to the GMIM model when only those subjects with tophi were considered.

\section{Achievement and maintenance of GMIM responses}

Subjects who had persistent lowering of urate during the RCT were followed into the OLE. As can be seen,
GMIM responses were maintained for the 2 years of the OLE (Fig. 5a, b). Moreover, patients who received placebo in the RCTs and were then switched to pegloticase $\mathrm{q} 2 \mathrm{w}$ in the OLE achieved GMIM responses comparable to those treated with biweekly pegloticase in the RCTs (Fig. 6a, b).

\section{Discussion}

The results from this analysis indicate that the GMIM criteria are useful as a composite outcome measure to capture response to pegloticase in chronic refractory gout patients. The measures comprising the composite outcome measure included $\mathrm{sU}$, tophus area, SJC, TJC, PGA, flares, and pain. The first five were based on a repeated measures mixed effects model with backward elimination [34] using data from the pegloticase RCTs; pain was added given the importance attributed to it by patients with gout $[36,37]$ and the fact that it was a component of the original remission criteria [33].

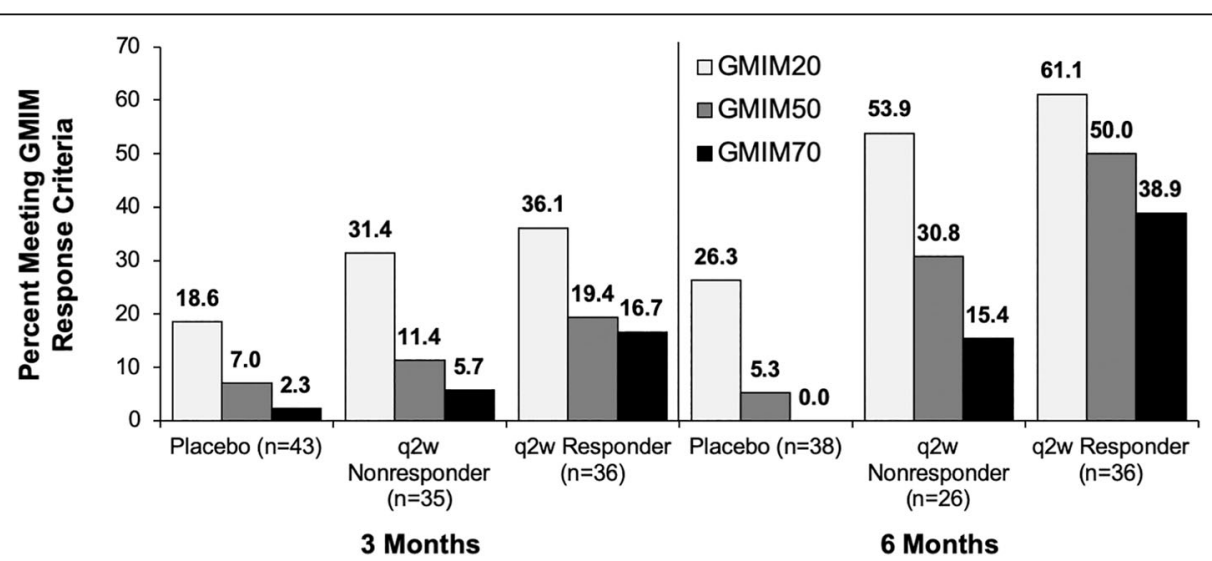

Fig. 3 Percentage of responders and nonresponders to pegloticase or placebo-treated subjects meeting GMIM criteria at 3 and 6 months. Data show percentage of subjects meeting GMIM criteria excluding serum urate $<6 \mathrm{mg} / \mathrm{dL}$. Number of evaluable subjects is shown for each group. The percentage of responders is shown above the bars. The statistical analysis of the data is shown in the supplementary material 


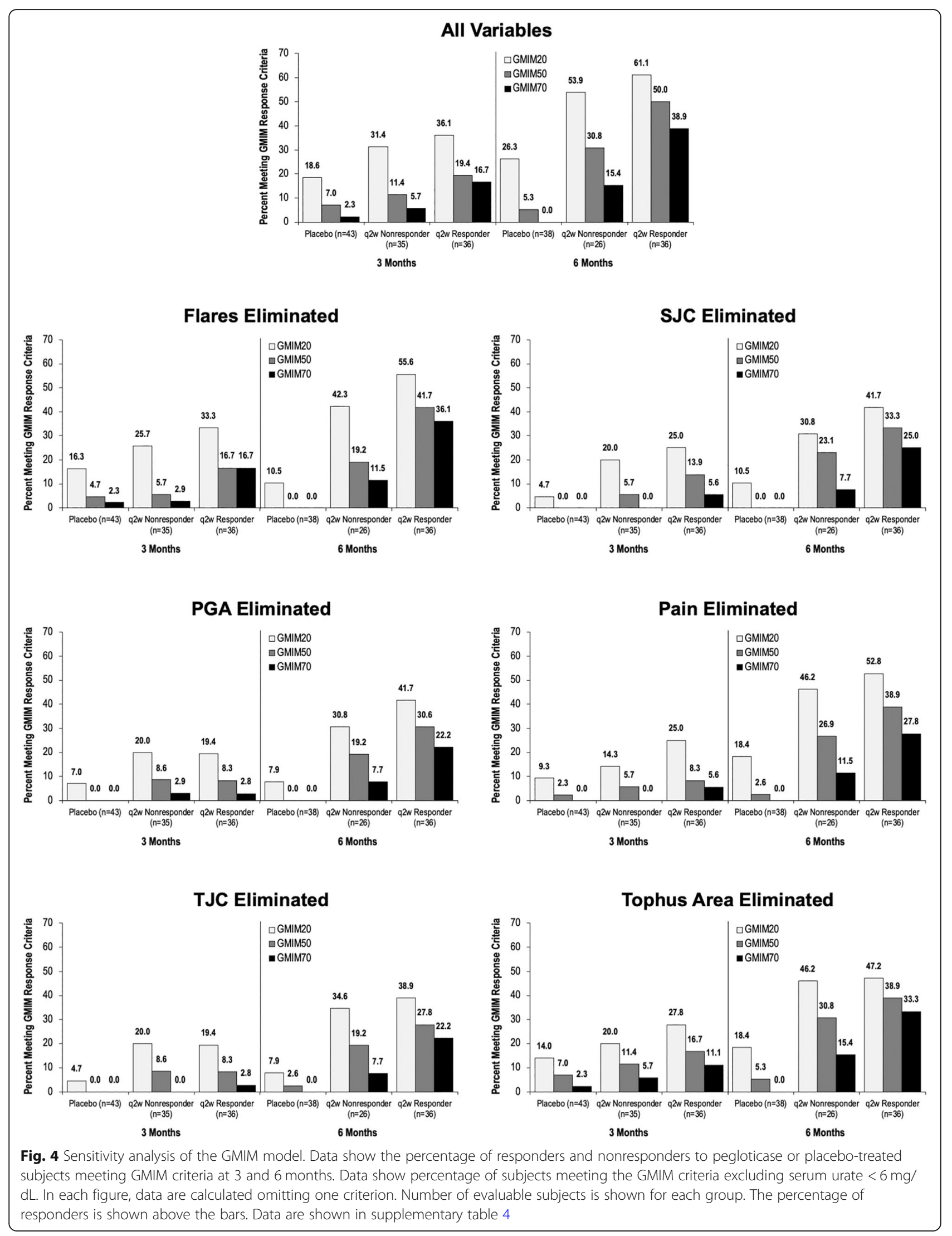



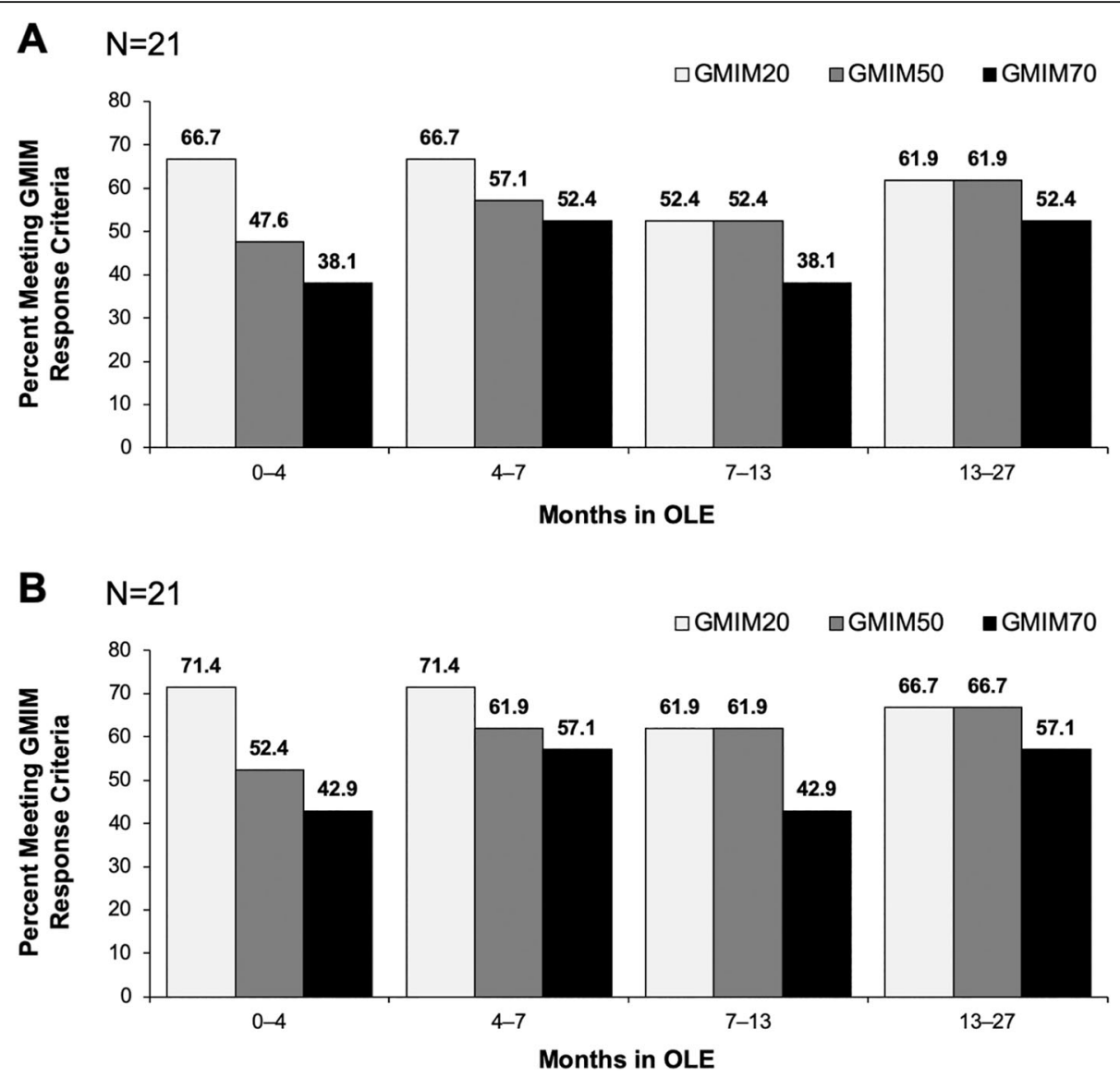

Fig. 5 Percentage of subjects with GMIM20, 50, and 70 responses during the OLE for patients treated with biweekly pegloticase in the RCT and OLE. a Results for all criteria including urate $<6.0 \mathrm{mg} / \mathrm{dL}$ and $\mathbf{b}$ Results for all GMIM criteria excluding urate $<6 \mathrm{mg} / \mathrm{dL}$

Inclusion of this endpoint into the composite GMIM is consistent with results from studies that have evaluated the importance of different symptoms in patients with acute or chronic gout $[16,30]$. Results from interviews of 30 patients with gout (10 with and 20 without clinically apparent tophi) indicated that pain was identified as being the cardinal, defining symptom of gout, leading to a range of impacts on health-related quality of life, most notably physical functioning and sleep [16]. Flares are included in the recommendations for elements in composite measures for assessment of gout treatment [28], the GAS composite [31], and proposed remission criteria [33], and this prompted their inclusion in the GMIM composite.

Results from the sensitivity analysis carried out in the present study showed that flares did not contribute significantly to the composite and that the contribution of pain was modest. This is not to suggest that flares are not important to patients, but just that they did not add information to the GMIM model. This could relate to the way information was collected in the RCTs. Flares were self-reported and pain was general and nonspecifically related to gout. More precise collection of these variables could add important information to a multivariable outcome model.

Evaluation of results eliminating the requirement for $\mathrm{sU}<6 \mathrm{mg} / \mathrm{dL}$ increased the percentages of patients achieving GMIM20, 50, and 70 among the $\mathrm{q} 2 \mathrm{w}$ subjects without persistent urate lowering and also resulted in small numbers of placebo-treated patients achieving these goals. The observation that the percentages of subjects without persistent urate lowering achieving GMIM20, 50, and 70 at 6 months is consistent with previous analyses of results from these studies indicating significant clinical improvements in these patients despite failure to achieve sustained urate lowering [38]. It has been suggested that these improvements may be related to the transient but profound reductions in urate observed in these patients [38]. Results from the OLE showed that GMIM responses were sustained in the patients who initiated pegloticase treatment in the RCT and continued in the OLE. They also demonstrated that switching from placebo to $\mathrm{q} 2 \mathrm{w}$ pegloticase treatment when progressing from the RCTs to the OLE resulted in clinical improvement comparable to that observed for 

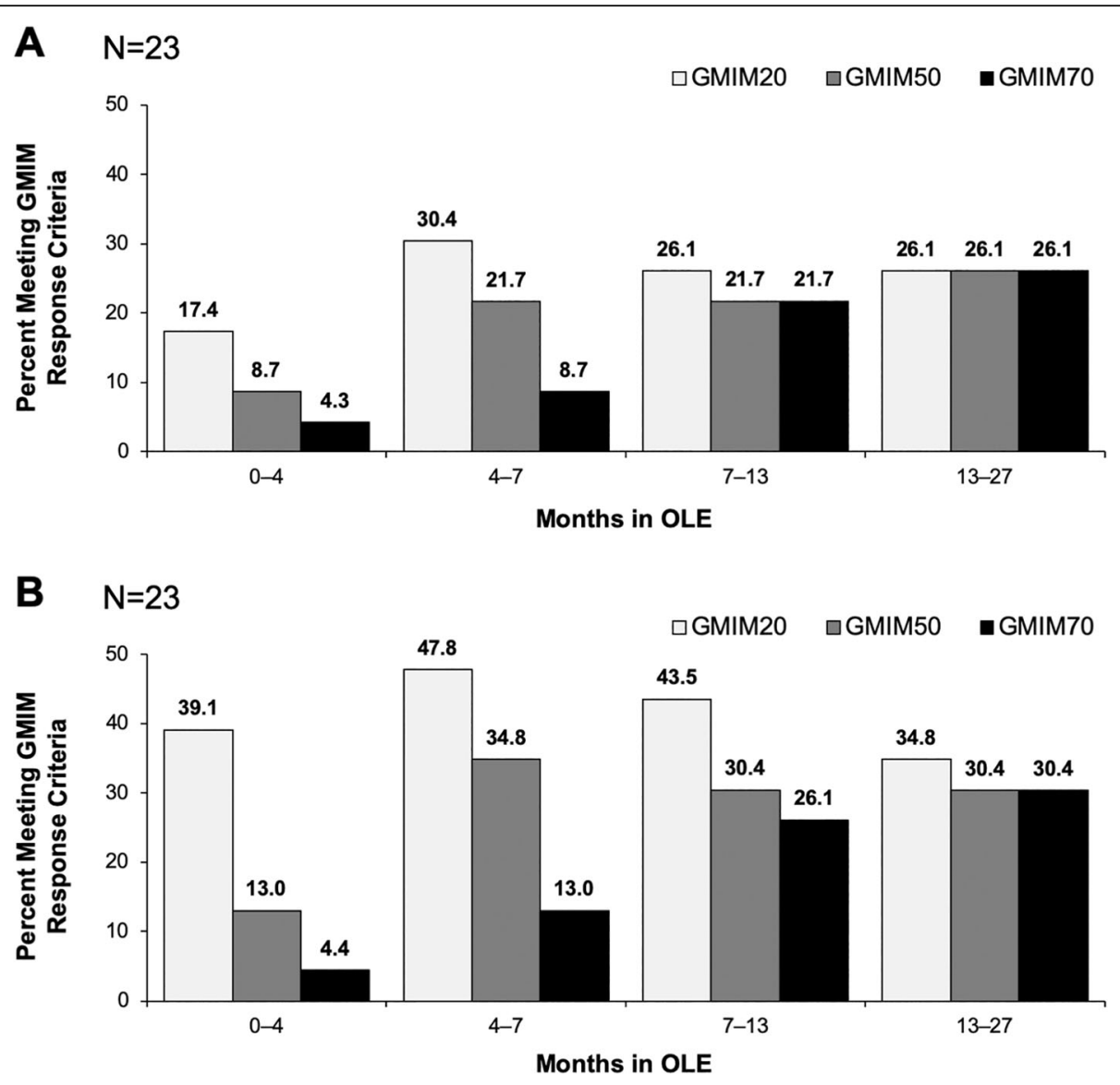

Fig. 6 Percentage of subjects with GMIM20, 50, and 70 responses during the OLE for patients who were treated with placebo in the RCT and biweekly pegloticase in the OLE. a Results for all GMIM criteria including urate $<6 \mathrm{mg} / \mathrm{dL}$ and $\mathbf{b}$ Results for all criteria excluding urate $<6.0 \mathrm{mg} / \mathrm{dL}$

those who initially received pegloticase and responded with persistent urate lowering in the RCTs and continued on this dose in the OLE. The ability of patients to "catch up" after delayed initiation of pegloticase should not be surprising since the patients randomized to placebo in the RCTs had gout symptoms for a mean of 13 years at the time of study entry [26]. Importantly, patients switching from placebo to pegloticase were not included in the generation of GMIM and, therefore, serve to validate the approach.

As noted above, a composite endpoint for assessment of gout treatment was developed before the GMIM. The GAS was based on longitudinal analysis of results from a multicenter observational cohort study (Kick-Off of the Italian Network for Gout [KING]), in which $68.7 \%$ of a cohort of 406 patients were treated with allopurinol and 13.6\% were treated with febuxostat [31]. A multistep process that began with all measures in the OMERACT core domain [20] and included factor analysis, linear discriminant analysis, and linear regression resulted in inclusion of four factors: gout flares in the past 12 months, $\mathrm{sU}$, pain, and number of tophi [31]. All of these measures were included in GMIM and both TJC and SJC were also added into GMIM, which differed from the GAS in that it was focused on responsivity to pegloticase treatment. Recently, Chinchilla et al. confirmed the predictive validity of the GAS, its correlation with the GIS, and its sensitivity to change [32]. It is important to note that the disease severity for patients enrolled in the KING study which provided the basis for the GAS was very different from those in the RCTs for pegloticase $[26,31]$. The patients in the pegloticase studies had longer duration of disease (12-16 years across treatment groups vs 3.8 years), greater numbers of tender (11.114.1 vs 1$)$ and swollen (8.9-13.2 vs 0$)$ joints, tophi (65.2\% to $76.7 \%$ vs $19.5 \%)$, flares ( $20-43$ over 18 months before treatment vs 0 over 3 months), and sU (9.4-10.4 $\mathrm{mg} / \mathrm{dL}$ vs $6.3 \mathrm{mg} / \mathrm{dL}$ ). In addition, patients enrolled in the pegloticase RCTs were required to have gout that was refractory to allopurinol treatment or to be intolerant of this medication whereas this was not the case in the KING study. GMIM was, therefore, tested in a very severe group of subjects with advanced gout. Whether it would be effective in subjects with less advanced disease 
remains to be evaluated, but the overlapping components of GAS and GMIM suggest that it might. A parallel effort to identify a multivariable outcome measure for acute gout might be worthwhile. Moreover, a direct comparison of the performance of GAS and GMIM might be informative.

There is an important limitation with respect to the GMIM as it is currently formulated. There is no adjustment for baseline variables with scores so low that the patient cannot achieve a specified $(20 \%, 50 \%$, or $70 \%)$ improvement regardless of the efficacy of treatment. This same problem applies to the American College of Rheumatology criteria for assessment of treatments for rheumatoid arthritis $[39,40]$ and has been used as a justification for composite measures that assess absolute disease activity vs change from baseline [40]. This limitation might be overcome by adjusting response criteria to include reduction of a given endpoint to 0 when baseline scores are sufficiently low. We addressed this issue by making the response criterion for a response improvement, 3 of the 5 GMIM parameters in addition to achieving $\mathrm{sU}<6 \mathrm{mg} / \mathrm{dL}$ and a decrease in flares. Finally, there is a general concern about composite criteria and whether they might overestimate responses has been articulated [41, 42] For this reason, GMIM was structured to have the 2 most frequently required components, decrease in $\mathrm{sU}<6.0 \mathrm{mg} / \mathrm{dL}$ and decrease in flares to be mandatory components.

\section{Conclusion}

In summary, the GMIM criteria effectively capture a change in disease severity in chronic refractory gout patients treated with pegloticase. It may serve as an evidence-based tool for assessment of therapies used to treat patients with similar baseline disease severity.

\section{Supplementary information}

Supplementary information accompanies this paper at https://doi.org/10. 1186/s13075-020-02254-4.

\section{Additional file 1. \\ Additional file 2. \\ Additional file 3. \\ Additional file 4. \\ Additional file 5.}

\section{Abbreviations}

GAS: Gout Activity Score; GIS: Gout Impact Scale; GMIM: Gout Multivariable Improvement Measure; HAQ: Health Assessment Questionnaire; KING: Kick-off of the Italian Network for Gout; OLE: Open-label extension;

OMERACT: Outcome Measures in Rheumatology; PGA: Patient global assessment; RCT: Randomized controlled trial; SJC: Swollen joint count: sU: Serum urate; TJC: Tender joint count; ULT: Urate-lowering therapy; VAS: Visual analog scale

Acknowledgements Not applicable.
Authors' contributions

All authors had full access to the study data, contributed to the writing and review of the manuscript, and approved the final version for publication.

\section{Funding}

This analysis was supported by Horizon Therapeutics, which did not influence the analysis or the conclusions reported in this communication.

\section{Availability of data and materials}

The data and analytic methods that support the findings of this study are available to qualified investigators by request to the corresponding author.

\section{Ethics approval and consent to participate}

The design of the two identical RCTs of pegloticase and their OLE that provided the data analyzed in this study (Clinicaltrials.gov identifiers: NCT00325195, NCT01356498) received institutional review board approval for each site, and written informed consent and Health Insurance Portability and Accountability Act assurances were completed for each participant prior to enrollment.

\section{Consent for publication}

Not applicable.

\section{Competing interests}

NS reports research grants from Pfizer and Amgen and advisory board/ consulting fees from Novartis, Horizon Therapeutics, Selecta Biosciences, Olatec, IFM Therapeutics, and Mallinckrodt Pharmaceuticals.

NLE reports consulting fees from Astra Zeneca, Horizon Therapeutics, Ironwood Pharmaceuticals, and SOBI International.

AEY reports consulting from Horizon Therapeutics.

PEL reports consulting fees from Horizon Therapeutics.

\section{Author details}

'Division of Rheumatology, Department of Medicine, Rutgers Robert Wood Johnson Medical School, New Brunswick, NJ, USA. ${ }^{2}$ Department of Medicine, University of Florida, Gainesville, FL, USA. ${ }^{3}$ Yeo Analytics, LLC, Jersey City, NJ, USA. ${ }^{4}$ AMPEL BioSolutions, LLC, Charlottesville, VA, USA.

Received: 23 March 2020 Accepted: 18 June 2020

Published online: 29 June 2020

\section{References}

1. Fransen J, van Riel PL. Outcome measures in inflammatory rheumatic diseases. Arthritis Res Ther. 2009;11:244.

2. Pincus T, Bergman MJ, Maclean R, Yazici Y. Complex measures and indices for clinical research compared with simple patient questionnaires to assess function, pain, and global estimates as rheumatology "vital signs" for usual clinical care. Rheum Dis Clin N Am. 2009:35:779-86.

3. Felson DT, Anderson JJ, Boers M, Bombardier C, Furst D, et al. American College of Rheumatology. Preliminary definition of improvement in rheumatoid arthritis. Arthritis Rheum. 1995;38:727-35.

4. Wells G, Becker JC, Teng J, Dougados M, Schiff M, et al. Validation of the 28joint Disease Activity Score (DAS28) and European League Against Rheumatism response criteria based on C-reactive protein against disease progression in patients with rheumatoid arthritis, and comparison with the DAS28 based on erythrocyte sedimentation rate. Ann Rheum Dis. 2009;68: 954-60.

5. Mumtaz A, Gallagher P, Kirby B, Waxman R, Coates LC, et al. Development of a preliminary composite disease activity index in psoriatic arthritis. Ann Rheum Dis. 2011;70:272-7.

6. FitzGerald O, Helliwell P, Mease P, Mumtaz A, Coates L, et al. Application of composite disease activity scores in psoriatic arthritis to the PRESTA data set. Ann Rheum Dis. 2012:71:358-62.

7. Helliwell PS, FitzGerald O, Fransen J, Gladman DD, Kreuger GG, et al. The development of candidate composite disease activity and responder indices for psoriatic arthritis (GRACE project). Ann Rheum Dis. 2013;72:986-91.

8. Bombardier C, Gladman DD, Urowitz MB, Caron D, Chang DH. Committee on Prognosis Studies in SLE Derivation of the SLEDAl: a disease activity index for lupus patients. Arthritis Rheum. 1992;35:630-40.

9. American College of Rheumatology Ad Hoc Committee on Systemic Lupus Erythematosus Response Criteria - The American College of Rheumatology: 
response criteria for systemic lupus erythematosus clinical trials: measures of overall disease activity. Arthritis Rheum. 2004:50:3418-3426.

10. Romero-Diaz J, Isenberg D, Ramsey-Goldman R. Measures of adult systemic lupus erythematosus: updated version of British Isles Lupus Assessment Group (BILAG 2004), European Consensus Lupus Activity Measurements (ECLAM), Systemic Lupus Activity Measure, Revised (SLAM-R), Systemic Lupus Activity Questionnaire for Population Studies (SLAQ), Systemic Lupus Erythematosus Disease Activity Index 2000 (SLEDAI-2K), and Systemic Lupus International Collaborating Clinics/American College of Rheumatology Damage Index (SDI). Arthritis Care Res (Hoboken). 2011;63(Suppl 11):S37-46.

11. Calin A, Nakache JP, Gueguen A, Zeidler H, Mielants H, Dougados M. Defining disease activity in ankylosing spondylitis: is a combination of variables (Bath Ankylosing Spondylitis Disease Activity Index) an appropriate instrument? Rheumatology (Oxford). 1999;38:878-82.

12. Bellamy N, Buchanan WW, Goldsmith CH, Campbell J, Stitt LW. Validation study of WOMAC: a health status instrument for measuring clinically important patient relevant outcomes to antirheumatic drug therapy in patients with osteoarthritis of the hip or knee. J Rheumatol. 1988;15:1833-40.

13. Lequesne M. Indices of severity and disease activity for osteoarthritis. Semin Arthritis Rheum. 1991;20(6 Suppl 2):48-54.

14. Vallejo MA, Rivera J, Esteve-Vives J, Rejas J, Group ICAF. A confirmatory study of the Combined Index of Severity of Fibromyalgia (ICAF*): factorial structure, reliability and sensitivity to change. Health Qual Life Outcomes. 2011:9:39.

15. Singh JA. The impact of gout on patient's lives: a study of African-American and Caucasian men and women with gout. Arthritis Res Ther. 2014;16:R132.

16. Tatlock S, Rüdell K, Panter C, Arbuckle R, Harrold LR, et al. What outcomes are important for gout patients? In-depth qualitative research into the gout patient experience to determine optimal endpoints for evaluating therapeutic interventions. Patient. 2017;10:65-79.

17. Grassi W, De Angelis R. Clinical features of gout. Reumatismo. 2012;63: 238-45.

18. Huang CF, Liu JC, Huang HC, Chuang SY, Chen Cl, Lin KC. Longitudinal transition trajectory of gouty arthritis and its comorbidities: a populationbased study. Rheumatol Int. 2017;37:313-22.

19. Schumacher HR, Edwards LN, Perez-Ruiz F, Becker M, Chen LX, et al. OMERACT 7 Special Interest Group. Outcome measures for acute and chronic gout. J Rheumatol. 2005;32:2452-5.

20. Schumacher HR, Taylor W, Edwards L, Grainger R, Schlesinger N, et al. Outcome domains for studies of acute and chronic gout. J Rheumatol. 2009:36:2342-5.

21. Becker MA, Schumacher HR, Espinoza LR, Wells AF, MacDonald P, et al. The urate-lowering efficacy and safety of febuxostat in the treatment of the hyperuricemia of gout: the CONFIRMS trial. Arthritis Res Ther. 2010;12:R63.

22. Huang $X, D u ~ H, ~ G u ~ J$, Zhao D, Jiang $L$, et al. An allopurinol-controlled, multicenter, randomized, double-blind, parallel between-group, comparative study of febuxostat in Chinese patients with gout and hyperuricemia. Int J Rheum Dis. 2014:17:679-86.

23. Tausche AK, Alten R, Dalbeth N, Kopicko J, Fung M, et al. Lesinurad monotherapy in gout patients intolerant to a xanthine oxidase inhibitor: a 6-month phase 3 clinical trial and extension study. Rheumatology (Oxford). 2017:56:2170-8.

24. Saag KG, Fitz-Patrick D, Kopicko J, Fung M, Bhakta N, et al. Lesinurad combined with allopurinol: a randomized, double-blind, placebo-controlled study in gout patients with an inadequate response to standard-of-care allopurinol (a US-based study). Arthritis Rheumatol. 2017;69:203-12.

25. Bardin T, Keenan RT, Khanna PP, Kopicko J, Fung M, et al. Lesinurad in combination with allopurinol: a randomised, double-blind, placebocontrolled study in patients with gout with inadequate response to standard of care (the multinational CLEAR 2 study). Ann Rheum Dis. 2017; 76:811-20.

26. Sundy JS, Baraf HS, Yood RA, Edwards NL, Gutierrez-Urena SR, et al. Efficacy and tolerability of pegloticase for the treatment of chronic gout in patients refractory to conventional treatment: two randomized controlled trials. JAMA. 2011;306:711-20.

27. Taylor TH, Mecchella JN, Larson RJ, Kerin KD, Mackenzie TA. Initiation of allopurinol at first medical contact for acute attacks of gout: a randomized clinical trial. Am J Med. 2012;125:1126-34.

28. Schumacher HR, Taylor W, Joseph-Ridge N, Perez-Ruiz F, Chen LX, et al. Outcome evaluations in gout. J Rheumatol. 2007;34:1381-5.
29. Taylor WJ, Singh JA, Saag KG, Dalbeth N, MacDonald PA, Edwards NL, et al. Bringing it all together: a novel approach to the development of response criteria for chronic gout clinical trials. J Rheumatol. 2011;38:1467-70.

30. Sarkin A, Gnanasakthy A, Lale R, Choi K, Hirsch J. A composite endpoint measure to consolidate multidimensional impact of treatment on gouty arthritis. Open J Rheumatol Autoimmune Dis. 2013;3:159-64.

31. Scirè CA, Carrara G, Viroli C, Cimmino MA, Taylor WJ, et al. Study Group for the Kick-Off of the Italian Network for Gout Study. Development and first validation of a disease activity score for gout. Arthritis Care Res (Hoboken). 2016;68:1530-7.

32. Chinchilla SP, Doherty M, Abhishek A. Gout Activity Score has predictive validity and is sensitive to change: results from the Nottingham Gout Treatment Trial (Phase II). Rheumatology (Oxford). 2019. https://doi.org/10 1093/rheumatology/key446 [Epub ahead of print].

33. de Lautour H, Taylor WJ, Adebajo A, Alten R, Burgos-Vargas R, et al. Development of preliminary remission criteria for gout using Delphi and 1000Minds consensus exercises. Arthritis Care Res (Hoboken). 2016; 68:667-72.

34. Schlesinger N, Edwards NE, Khanna P, Yeo A, Lipsky PE. Evaluation of proposed criteria for evidence based development of criteria for complete response in patients with chronic refractory gout. ACR Open Rheumatology. 2019. https://doi.org/10.1002/acr2.1025.

35. Becker MA, Baraf HS, Yood RA, Dillon A, Vázquez-Mellado J, et al. Long-term safety of pegloticase in chronic gout refractory to conventional treatment. Ann Rheum Dis. 2013;72:1469-74.

36. Singh JA, Taylor WJ, Simon LS, Khanna PP, Stamp LK, FM MQ, et al. Patientreported outcomes in chronic gout: a report from OMERACT 10. J Rheumatol. 2011;38:1452-7.

37. Shields GE, Beard SM. A systematic review of the economic and humanistic burden of gout. Pharmacoeconomics. 2015;33:1029-47.

38. Mandell BF, Yeo A, Lipsky PE. Tophus resolution in patients with chronic refractory gout who have persistent urate-lowering responses to pegloticase. Arthritis Res Ther. 2018;20(1):286.

39. Smolen JS, Breedveld FC, Schiff MH, Kalden JR, Emery P, et al. A simplified disease activity index for rheumatoid arthritis for use in clinical practice. Rheumatology (Oxford). 2003;42:244-57.

40. van Riel PL, van Gestel AM. Clinical outcome measures in rheumatoid arthritis. Ann Rheum Dis. 2000;59(Suppl 1):i28-31.

41. Freemantle N, Calvert M, Wood J, Eastaugh J, Griffin C. Composite outcomes in randomized trials: greater precision but with greater uncertainty? JAMA. 2003;289(19):2554-9.

42. Cordoba G, Schwartz L, Woloshin S, Bae H, Gøtzsche PC. Definition, reporting, and interpretation of composite outcomes in clinical trials: systematic review. BMJ. 2010;341:c3920.

\section{Publisher's Note}

Springer Nature remains neutral with regard to jurisdictional claims in published maps and institutional affiliations.

\section{Ready to submit your research? Choose BMC and benefit from:}

- fast, convenient online submission

- thorough peer review by experienced researchers in your field

- rapid publication on acceptance

- support for research data, including large and complex data types

- gold Open Access which fosters wider collaboration and increased citations

- maximum visibility for your research: over $100 \mathrm{M}$ website views per year

At $\mathrm{BMC}$, research is always in progress.

Learn more biomedcentral.com/submission 